\title{
Modelling Alcoholism as a Contagious Disease: A Mathematical Model with Awareness Programs and Time Delay
}

\author{
Shuang-Hong Ma, ${ }^{1,2}$ Hai-Feng Huo, ${ }^{1,2}$ and Xin-You Meng ${ }^{2,3}$ \\ ${ }^{1}$ College of Electrical and Information Engineering, Lanzhou University of Technology, Lanzhou 730050, China \\ ${ }^{2}$ Institute of Applied Mathematics, Lanzhou University of Technology, Lanzhou 730050, China \\ ${ }^{3}$ Institute of Systems Science, Northeastern University, Shenyang 110819, China \\ Correspondence should be addressed to Hai-Feng Huo; hfhuo@lut.cn
}

Received 8 August 2015; Accepted 10 September 2015

Academic Editor: Luca Guerrini

Copyright (C) 2015 Shuang-Hong Ma et al. This is an open access article distributed under the Creative Commons Attribution License, which permits unrestricted use, distribution, and reproduction in any medium, provided the original work is properly cited.

A dynamic alcohol consumption model with awareness programs and time delay is formulated and analyzed. The aim of this model is to capture the effects of awareness programs and time delay in controlling the alcohol problems. We introduce awareness programs by media in the model as a separate class with growth rate of the cumulative density of them being proportional to the number of mortalities induced by heavy drinking. Susceptible population will isolate themselves and avoid contact with the heavy drinkers or become aware of risk of heavy drinking and decline to drink due to such programs. In particular, we incorporate time delay because the nonconsumer population will take a period of time to become an alcohol consumer. We find that the model has two equilibria: one without alcohol problems and one where alcohol problems are endemic in population. The model analysis shows that though awareness programs cannot eradicate alcohol problems, they are effective measures in controlling the alcohol problems. Further, we conclude that the time delay in alcohol consumption habit which develops in susceptible population may result in Hopf bifurcation by increasing the value of time delay. Some numerical simulation results are also given to support our theoretical predictions.

\section{Introduction}

Alcoholism, also known as alcohol dependence, is a function of social epidemic, environmental contexts, individuals' preferences, and family history. Alcohol consumption has been identified as a major contributor to the global burden of chronic disease, injury, and economic cost [1-3]. Alcohol abuse can also lead to a range of negative social effects such as violence and antisocial and criminal behavior. The World Health Organization reports that the harmful use of alcohol causes approximately 3.3 million deaths every year (or 5.9\% of all the global deaths), and $5.1 \%$ of the global burden of disease is attributable to alcohol consumption [4]. Therefore, alcoholism is among the main targeted health risk behaviors due to the high relevance of negative health and social effects. The study of alcoholism has become an important aspect of social epidemic.
The spread of health risk behavior within a community can be viewed as a diffusion process with its own incidence rate. In this situation, the social interaction is considered to be the key factor in spreading the behavior which can result in adverse health effects. For this reason, alcoholism can be viewed as a treatable contagious disease. Mathematical model is a predictive tool which can mimic the process of infectious diseases and provide useful measures to analyze the spread and control of infectious diseases [5, 6]. Furthermore, mathematical models for human behaviors related to addictions have been developed from epidemiological models for the spread of infectious diseases, including drinking, smoking, and drug use (see [7-10] and the references contained therein). In particular, numerous mathematical models for alcohol problems have been formulated and studied recently [11-21]. Benedict [11] modelled alcoholism as a contagious disease and studied how "infected" drinking buddies spread 
problem drinking. Manthey et al. [12] studied campus drinking and suggested that the reproductive numbers are not sufficient to predict whether drinking behavior will persist on campus and that the pattern of recruiting new members plays a significant role in the reduction of campus alcohol problems. Environmental and peer influences combined to create a culture of drinking were studied in [13-16]. In addition, the two-stage models (one stage where people admit having alcohol problem and another stage where people do not admit having alcohol problem) have been developed in [17, 18]. Bhunu and Mushayabasa [19] studied the cointeraction of alcoholism and smoking in a community. Walters et al. [20] also discussed alcohol problems, and their results showed that an increase in the recovery rate decreased the proportion of binge drinkers in the population. Wang et al. [21] presented a deterministic SATQ-type mathematical model (the model including susceptible, alcoholism, treating, and quitting drinking compartments) for the spread of alcoholism with two control strategies to gain insights into this increasingly concerned-about health and social phenomenon. The optimal control strategies are derived by proposing an objective functional and using Pontryagin's Maximum Principle.

A rational step is to make people aware of the alcohol problems through the media. Media (e.g., Radio, Newspapers, Billboards, TV, and Internet), being the prime source of information, can not only influence the individuals' behavior but also increase the governmental health care involvement to control the spread of heavy drinking. These behavioral responses can change the transmission patterns and declination to drink. In view of this, there is a need to incorporate the effect of awareness programs through the media in the mathematical models. In recent years, many mathematical models have been used for studying the impact of awareness programs by media on epidemic outbreaks (see [22-32] and the references cited therein). These studies suggested that education and media have a huge impact on controlling the spread of infectious diseases. However, most of these studies assumed that the media reduced the contact rate of susceptible with infective individuals. In addition, these studies assumed that the cumulative density of awareness programs is constant in models. Hence, it is more reasonable to consider that the cumulative density of awareness programs varies. Misra et al. [33] have explicitly incorporated the cumulative density of awareness programs in the modelling process, which is a separate dynamic variable whose growth depends on the size of epidemic. The results showed that awareness programs by media are implemented in accordance with the size of infective population. Considering the effect of susceptible population influenced by media to be limited, Misra et al. [34] have considered interaction between susceptible population and awareness programs as Holling-II functional response function.

Recently, Huo and Wang [35] have studied drinking dynamics and focused on awareness programs and treatment in the modelling process. They have extended the model in [33] via including a treatment class and established some sufficient conditions for the stability of the alcohol-free and alcohol-present equilibria.
It is well known that delay differential equations exhibit much more complicated dynamics than ordinary differential equations since the time delay may affect the stability of the system and even lead to instability, oscillation, or bifurcation phenomena [34, 36-39]. In most of the above studies, with susceptible individual contacts with heavy drinkers, he/she will become a heavy drinker at once. However, the alcoholism epidemic should consider the effects of the time delay. Since a nondrinker or moderate drinker is infected by heavy drinkers, there is a time delay during which a susceptible individual becomes heavy drinker. Hence, it is more realistic to consider the time delay in the modelling alcoholism process.

In this paper, motivated by the above works, we present a nonlinear alcoholism model with awareness programs and time delay. First, we assume that the growth rate of the cumulative density of awareness programs is proportional to the number of mortalities induced by heavy drinking. Hence, the awareness about drinking will alert the susceptible individuals so that they isolate themselves and decline to drink or drink moderately (small intake of alcohol may be beneficial to health). Second, we assume that the heavy drinkers can recover from heavy alcohol drinking due to counselling, health reasons, treatment, prohibition, tax hiked on alcohol beverages, and so forth. A fraction of recovered population will join the unaware susceptible population whereas the rest will join the aware population. In particular, we incorporate time delay $\tau$ to describe the time needed for a susceptible individual to become a heavy alcohol user. The goal is to analyze the impact of awareness programs and time delay on the alcohol drinking distribution. We also analyze the effect of time delay on system stability. Our results show that awareness programs are an effective measure in controlling heavy drinking. Our results also show that the system will change its stability with the increase of the value of time delay.

The remainder of this paper is organized as follows. In Section 2, we formulate the delayed mathematical model. The condition for the existence of the equilibria is shown in Section 3. In Section 4, we discuss the stability of equilibria and the existence of Hopf bifurcation. To support our theoretical predictions, some numerical simulations are included in Section 5. A brief conclusion is also given in Section 6.

\section{Model Formulation}

The total population in the model is $P(t)$ at time $t$, and it is divided into three types: $S(t), X(t)$, and $A(t) . S(t)$ denotes susceptible individuals who do not drink or drink only moderately. $A(t)$ is referred to as heavy drinkers. The class $X(t)$ is aware population who are aware of risk and decline to drink or drink moderately. It is supposed that the cumulative density of awareness programs driven by media at time $t$ is $M(t)$. The growth rate of $M(t)$ is proportional to the number of mortalities induced by heavy drinking. It is also assumed that heavy drinking only spreads due to the direct contact between susceptible individuals and heavy drinkers. Susceptible population avoid being in contact with the heavy drinkers because the implementation of awareness programs results in awareness about heavy drinking. In view of this, a separate class $X(t)$ is formed. It is assumed that when 


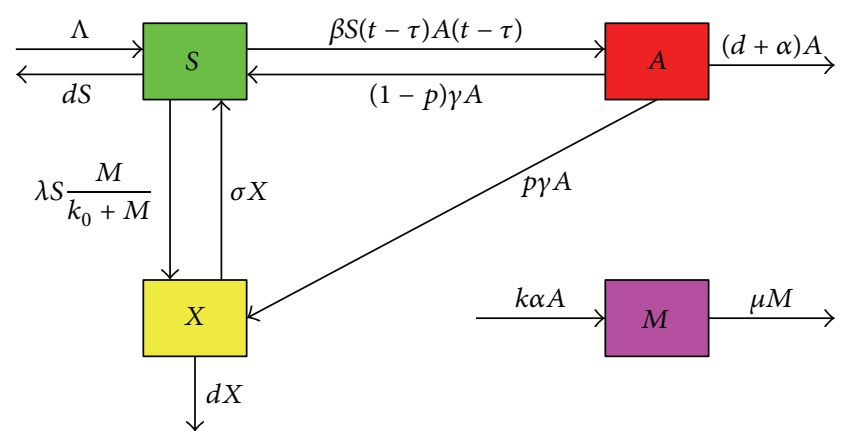

Figure 1: The transfer diagram of system (1).

a nondrinker or moderate drinker is "infected" by heavy drinkers, there is a time delay $\tau$ during which the alcohol consumption habit develops in his/her body. It is only after such time delay $\tau$ that a susceptible individual becomes a heavy drinker.

Considering homogenous mixing, the transfer diagram is shown in Figure 1.

The transfer diagram leads to the following dynamic alcohol consumption model with awareness programs and time delay:

$$
\begin{aligned}
\frac{\mathrm{d} S(t)}{\mathrm{d} t}= & \Lambda-\beta S(t-\tau) A(t-\tau)-\lambda S(t) \frac{M(t)}{k_{0}+M(t)} \\
& +\sigma X(t)+(1-p) \gamma A(t)-d S(t), \\
\frac{\mathrm{d} A(t)}{\mathrm{d} t}= & \beta S(t-\tau) A(t-\tau)-(\gamma+d+\alpha) A(t), \\
\frac{\mathrm{d} X(t)}{\mathrm{d} t}= & \lambda S(t) \frac{M(t)}{k_{0}+M(t)}+p \gamma A(t)-(d+\sigma) X(t), \\
\frac{\mathrm{d} M(t)}{\mathrm{d} t}= & k \alpha A(t)-\mu M(t),
\end{aligned}
$$

where $\Lambda$ is the recruitment rate of susceptible individuals who do not drink or drink only moderately, $\beta$ is the transmission coefficient of the infection for the susceptible individuals from the heavy drinkers, $\lambda$ is the dissemination rate of awareness programs among susceptible population, $k_{0}$ limits the effect of awareness programs on susceptible population, known as half saturation point for interactions of HollingII functional response function, $\sigma$ is the transmission rate of transferring from aware population to unaware susceptible population due to fading of memory or certain factors, and so forth, $\gamma$ is the recovery rate of heavy drinkers, $p$ represents fraction of recovered individuals joining aware class, $1-p$ represents fraction of recovered individuals joining unaware susceptible class, $d$ is the natural death rate of the general population in the model, $\alpha$ is the death rate due to heavy alcohol consumption, $k$ is the implementation rate of awareness programs, $\mu$ is the depletion rate of awareness programs due to ineffectiveness such as social and psychological factors in population, and $\tau$ is the time lag due to development of alcohol consumption habit.
All the above parameters are assumed to be positive constants. We suppose that the initial condition for system (1) takes the form $S(\theta), A(\theta), X(\theta) \geq 0, \theta \in[-\tau, 0]$, and $S(0), A(0), X(0)>0$, where $S(\theta), A(\theta), X(\theta) \in C\left([-\tau, 0], R_{+}\right)$, $R_{+}=\{x \mid x \geq 0\}$, and $M(0)=M_{0} \geq 0$.

Using the fact that $P(t)=S(t)+A(t)+X(t)$, system (1) is rewritten as

$$
\begin{aligned}
& \frac{\mathrm{d} A(t)}{\mathrm{d} t} \\
& =\beta[P(t-\tau)-A(t-\tau)-X(t-\tau)] A(t-\tau) \\
& \quad-(\gamma+d+\alpha) A(t), \\
& \frac{\mathrm{d} X(t)}{\mathrm{d} t} \\
& =\lambda[P(t)-A(t)-X(t)] \frac{M(t)}{k_{0}+M(t)}+P \gamma A(t) \\
& \quad-(d+\sigma) X(t), \\
& \frac{\mathrm{d} P(t)}{\mathrm{d} t}=\Lambda-d P(t)-\alpha A(t), \\
& \frac{\mathrm{d} M(t)}{\mathrm{d} t}=k \alpha A(t)-\mu M(t) .
\end{aligned}
$$

Since system (1) is equivalent to system (2a), (2b), (2c), and $(2 \mathrm{~d})$, we study system (2a), (2b), (2c), and (2d) in this paper. From (2c), we can obtain

$$
\lim _{t \rightarrow \infty} \sup P(t) \leq \frac{\Lambda}{d}
$$

then

$$
\begin{aligned}
& 0 \leq A \\
& X \leq P \leq \frac{\Lambda}{d} .
\end{aligned}
$$

From (2d), we have

$$
\lim _{t \rightarrow \infty} \sup M(t) \leq \frac{k \alpha \Lambda}{\mu d} .
$$

Hence, the feasible region of system (2a), (2b), (2c), and (2d) is given by the set

$$
\begin{aligned}
\Omega & =\left\{(A, X, P, M) \in R_{+}^{4}: 0 \leq A, X \leq P \leq \frac{\Lambda}{d}, 0 \leq M\right. \\
& \left.\leq \frac{k \alpha \Lambda}{\mu d}\right\},
\end{aligned}
$$

which is positively invariant with respect to system (2a), (2b), (2c), and (2d). We will consider dynamic behavior of system (2a), (2b), (2c), and (2d) on the set $\Omega$.

\section{Existence of Equilibria}

It is obvious that system (2a), (2b), (2c), and (2d) has one alcohol-free equilibrium $E_{0}(0,0, \Lambda / d, 0)$ and one alcoholpresent equilibrium $E_{1}\left(A^{*}, X^{*}, P^{*}, M^{*}\right)$. The existence of 
equilibrium $E_{0}$ is trivial. In the following, we will prove the existence of alcohol-present equilibrium $E_{1}$.

The basic reproduction number of the alcohol consumption model can be easily obtained by the next generation matrix method formulated in [40], which is given by the following expression:

$$
R_{0}=\frac{\beta \Lambda}{d(\gamma+d+\alpha)},
$$

and it acts as a threshold as is shown in the following result.

Theorem 1. System (2a), (2b), (2c), and (2d) always has the alcohol-free equilibrium $E_{0}(0,0, \Lambda / d, 0)$. Furthermore, system (2a), (2b), (2c), and (2d) has a unique alcohol-present equilibrium $E_{1}\left(A^{*}, X^{*}, P^{*}, M^{*}\right)$ if $R_{0}>1$.

Proof. For equilibrium $E_{1}, A^{*}, X^{*}, P^{*}$, and $M^{*}$ satisfy the following algebraic equations:

$$
\begin{aligned}
& \beta\left(P^{*}-A^{*}-X^{*}\right) A^{*}-(\gamma+d+\alpha) A^{*}=0, \\
& \lambda\left(P^{*}-A^{*}-X^{*}\right) \frac{M^{*}}{k_{0}+M^{*}}+p \gamma A^{*}-(d+\sigma) X^{*} \\
& \quad=0, \\
& \Lambda-d P^{*}-\alpha A^{*}=0, \\
& k \alpha A^{*}-\mu M^{*}=0 .
\end{aligned}
$$

From (8c), we obtain

$$
P^{*}=\frac{\Lambda-\alpha A^{*}}{d}
$$

The fourth equation (8d) yields

$$
M^{*}=\frac{k \alpha}{\mu} A^{*} .
$$

Substituting (9) into (8a), we get

$$
X^{*}=\frac{\Lambda-\alpha A^{*}-d A^{*}}{d}-\frac{\gamma+d+\alpha}{\beta} .
$$

Then, substituting (9)-(11) into (8b), we have a quadratic equation of $A^{*}$ as follows:

$$
a_{1}\left(A^{*}\right)^{2}+a_{2} A^{*}+a_{3}=0
$$

where

$$
\begin{aligned}
a_{1}= & \alpha k\left(p \gamma+\alpha+d+\frac{\alpha \sigma}{d}+\sigma\right), \\
a_{2}= & \frac{\alpha k(\gamma+d+\alpha)(\lambda+d+\sigma)}{\beta} \\
& +\mu k_{0}\left(p \gamma+\alpha+d+\frac{\alpha \sigma}{d}+\sigma\right)-\alpha k \Lambda\left(1+\frac{\sigma}{d}\right), \\
a_{3}= & \mu k_{0}(d+\sigma)\left(\frac{\gamma+d+\alpha}{\beta}-\frac{\Lambda}{d}\right) \\
= & \frac{\mu k_{0}(d+\sigma)(\gamma+d+\alpha)}{\beta}\left(1-R_{0}\right) .
\end{aligned}
$$

It is apparent that the existence of equilibrium $E_{1}$ is equivalent to the existence of $A^{*}$. Obviously, $a_{1}>0$, and $a_{3}<0$ holds naturally when $R_{0}>1$. Hence, (12) has a unique positive solution, say $A^{*}$. Using the value of $A^{*}$, we can get positive solutions $P^{*}, M^{*}$, and $X^{*}$, respectively. So system (2a), (2b), (2c), and (2d) has a unique alcohol-present equilibrium $E_{1}$ if $R_{0}>1$. This completes the proof.

\section{Stability Analysis}

In this section, we will investigate the stability of the equilibria of system (2a), (2b), (2c), and (2d).

4.1. Stability Analysis with $\tau=0$. First, we investigate the stability under the condition of $\tau=0$. The stability of two equilibria $E_{0}$ and $E_{1}$ of system (2a), (2b), (2c), and (2d) can be given in the following theorem.

Theorem 2. For $\tau=0$, one has the following results:

(i) The alcohol-free equilibrium $E_{0}$ is globally asymptotically stable if $R_{0}<1$.

(ii) The alcohol-free equilibrium $E_{0}$ is unstable and the alcohol-present equilibrium $E_{1}$ exists if $R_{0}>1$.

Furthermore, equilibrium $E_{1}$ is locally asymptotically stable if the coefficients of the characteristic equation of system (2a), (2b), (2c), and (2d) at $E_{1}$ satisfy the Routh-Hurwitz criterion.

Proof. When $\tau=0$, the Jacobian matrix $J$ of system (2a), (2b), $(2 \mathrm{c})$, and $(2 \mathrm{~d})$ is

$$
J=\left(\begin{array}{cccc}
\beta(P-2 A-X)-(\gamma+d+\alpha) & -\beta A & \beta A & 0 \\
p \gamma-\frac{\lambda M}{k_{0}+M} & -\frac{\lambda M}{k_{0}+M}-(d+\sigma) & \frac{\lambda M}{k_{0}+M} & \frac{\lambda k_{0}(P-A-X)}{\left(k_{0}+M\right)^{2}} \\
-\alpha & 0 & -d & 0 \\
\alpha k & 0 & 0 & -\mu
\end{array}\right) .
$$


(i) For the alcohol-free equilibrium $E_{0}(0,0, \Lambda / d, 0)$, the matrix $J$ at $E_{0}$ can be written as

$$
\begin{aligned}
& J_{0}=\left.J\right|_{E_{0}} \\
& =\left(\begin{array}{cccc}
\frac{\beta \Lambda}{d}-(\gamma+d+\alpha) & 0 & 0 & 0 \\
p \gamma & -(d+\sigma) & 0 & \frac{\lambda \Lambda}{d+k_{0}} \\
-\alpha & 0 & -d & 0 \\
\alpha k & 0 & 0 & -\mu
\end{array}\right) .
\end{aligned}
$$

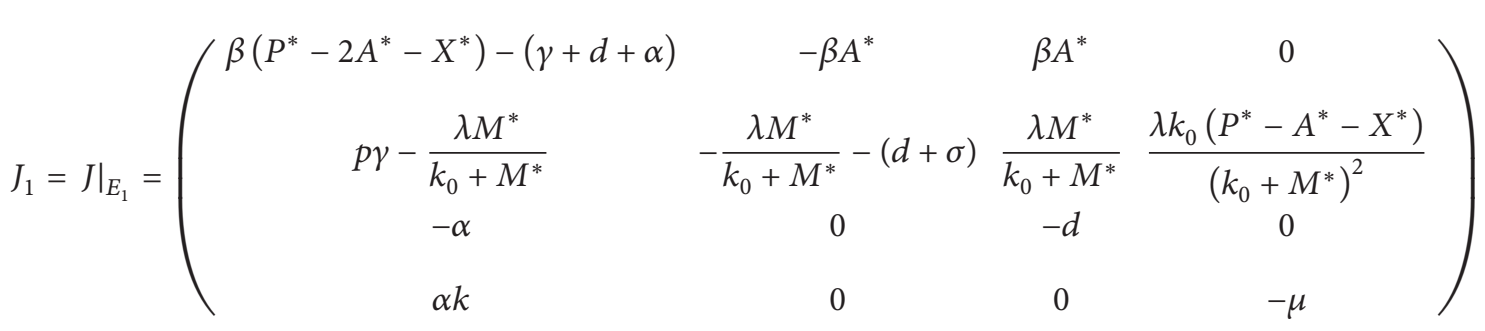

$$
\begin{aligned}
& =\left(\begin{array}{cccc}
J_{11} & J_{12} & J_{13} & 0 \\
J_{21} & J_{22} & J_{23} & J_{24} \\
J_{31} & 0 & J_{33} & 0 \\
J_{41} & 0 & 0 & J_{44}
\end{array}\right) .
\end{aligned}
$$

The characteristic equation of system (2a), (2b), (2c), and (2d) at $E_{1}$ is $\operatorname{det}\left(\varphi I-J_{1}\right)=0$, where $I$ is the identity matrix of size 4 and $\varphi$ is the eigenvalue of matrix $J_{1}$. By simple computation, the characteristic equation can be expressed:

$$
\varphi^{4}+b_{1} \varphi^{3}+b_{2} \varphi^{2}+b_{3} \varphi+b_{4}=0
$$

where

$$
\begin{aligned}
b_{1}= & -\left(J_{11}+J_{22}+J_{33}+J_{44}\right) \\
b_{2} & =J_{11} J_{22}+J_{11} J_{33}+J_{11} J_{44}+J_{22} J_{33}+J_{22} J_{44}+J_{33} J_{44} \\
& -\left(J_{12} J_{21}+J_{13} J_{31}\right) \\
b_{3} & =J_{12} J_{21} J_{33}+J_{12} J_{21} J_{44}+J_{13} J_{31} J_{22}+J_{13} J_{31} J_{44} \\
& -\left(J_{11} J_{22} J_{33}+J_{11} J_{22} J_{44}+J_{11} J_{33} J_{44}+J_{22} J_{33} J_{44}\right. \\
& \left.+J_{12} J_{24} J_{41}+J_{12} J_{31} J_{23}\right) \\
b_{4} & =J_{11} J_{22} J_{33} J_{44}+J_{12} J_{23} J_{31} J_{44}+J_{12} J_{24} J_{33} J_{41} \\
& -J_{12} J_{21} J_{33} J_{44}-J_{13} J_{22} J_{31} J_{44} .
\end{aligned}
$$

Notice that $R_{0}>1$; it can be calculated that the coefficients $b_{i}(i=1,2,3,4$. $)$ all are positive according to $J_{i i}$ $(i=1,2,3,4$. $)$ and $J_{12}, J_{31}$ all are negative and $J_{13}, J_{21}, J_{23}$, $J_{24}$, and $J_{41}$ all are positive. Furthermore, by using the RouthHurwitz criterion [41], it is easy to see that the eigenvalues of the above characteristic equation have negative real parts if the conditions

$$
\begin{array}{r}
b_{1} b_{2}-b_{3}>0 \\
b_{1} b_{2} b_{3}-b_{3}^{2}-b_{1}^{2} b_{4}>0
\end{array}
$$

hold. This shows that the alcohol-present equilibrium $E_{1}$ is locally asymptotically stable, and the proof is complete.

Moreover, we know that $R_{0}$ is a threshold value which determines whether alcohol problems will die out or become endemic in the population. The alcohol-free equilibrium $E_{0}$ undergoes a transcritical bifurcation at $R_{0}=1$. We are now interested in knowing which model parameter has the greatest effect on $R_{0}$ and hence has the greatest effect in controlling the alcohol consumption.

Using the approach in Chitnis et al. [42], we calculate the normalized forward sensitivity indices of $R_{0}$. Let

$$
\Upsilon_{q}^{R_{0}}=\frac{\partial R_{0}}{\partial q} \times \frac{q}{R_{0}}
$$




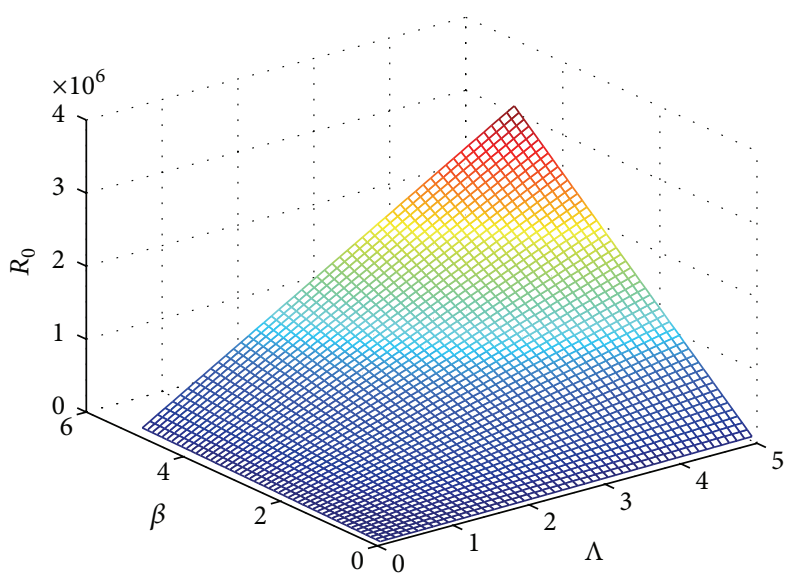

Figure 2: The effect of increasing $\beta$ and $\Lambda$ on $R_{0}$.

denote the sensitivity index of $R_{0}$ with respect to the parameter $q$. We get

$$
\begin{aligned}
& \Upsilon_{\beta}^{R_{0}}=\Upsilon_{\Lambda}^{R_{0}}=+1, \\
& \Upsilon_{d}^{R_{0}}=-\left(1+\frac{d}{\gamma+d+\alpha}\right), \\
& \Upsilon_{\gamma}^{R_{0}}=-\frac{\gamma}{\gamma+d+\alpha}, \\
& \Upsilon_{\alpha}^{R_{0}}=-\frac{\alpha}{\gamma+d+\alpha} .
\end{aligned}
$$

Considering that the natural death rate $d$ is a relatively stable constant, we find that $\Upsilon_{\beta}^{R_{0}}$ and $\Upsilon_{\Lambda}^{R_{0}}$ are the largest sensitivity indexes. We focus on reducing the value of $\beta$ or $\Lambda$ whose reduction by $1 \%$ would reduce $R_{0}$ by $1 \%$. In contrast, there is a need to increase $\gamma$ in order to control alcohol problems. In particular, increasing $\alpha$ results in promoting the dissemination rate $\lambda$ of awareness programs; hence, the value of $R_{0}$ decreases. Therefore, media coverage for the negative effects and the number of mortalities induced by heavy drinking is very important. At the same time, this sensitivity analysis tells us that the efforts through awareness programs to increase prevention, which cause the number of aware populations $X(t)$ to increase, are effective in controlling the spread of habitual alcohol consumption. To clarify this, the numerical simulation results will be also performed in Section 5 (see Figures 2 and 3).

4.2. Stability Analysis with $\tau>0$. In this subsection, we will be concerned with the effect of time delay on the stability of the alcohol-present equilibrium $E_{1}$. Let $u(t)=$ $(a(t), x(t), p(t), m(t))^{T}$; by linearizing system (2a), (2b), (2c), and $(2 \mathrm{~d})$ around the alcohol-present equilibrium $E_{1}$, we have

$$
\frac{d u}{d t}=B u(t)+C u(t-\tau)
$$

where

$$
\begin{aligned}
& B=\left(\begin{array}{cccc}
-(\gamma+d+\alpha) & 0 & 0 & 0 \\
p \gamma-\frac{\lambda M^{*}}{k_{0}+M^{*}} & -\frac{\lambda M^{*}}{k_{0}+M^{*}}-(d+\sigma) & \frac{\lambda M^{*}}{k_{0}+M^{*}} & \frac{\lambda k_{0}\left(P^{*}-A^{*}-X^{*}\right)}{\left(k_{0}+M^{*}\right)^{2}} \\
-\alpha & 0 & -d
\end{array}\right)=\left(\begin{array}{cccc}
b_{11} & 0 & 0 & 0 \\
b_{21} & b_{22} & b_{23} & b_{24} \\
b_{31} & 0 & b_{33} & 0 \\
b_{41} & 0 & 0 & b_{44}
\end{array}\right) \\
& C=\left(\begin{array}{crrr}
\gamma+d+\alpha-\beta A^{*} & -\beta A^{*} & \beta A^{*} & 0 \\
0 & 0 & 0 & 0 \\
0 & 0 & 0 & 0 \\
0 & 0 & 0 & 0
\end{array}\right)=\left(\begin{array}{rrrr}
c_{11} & c_{12} & c_{13} & 0 \\
0 & 0 & 0 & 0 \\
0 & 0 & 0 & 0 \\
0 & 0 & 0 & 0
\end{array}\right)
\end{aligned}
$$



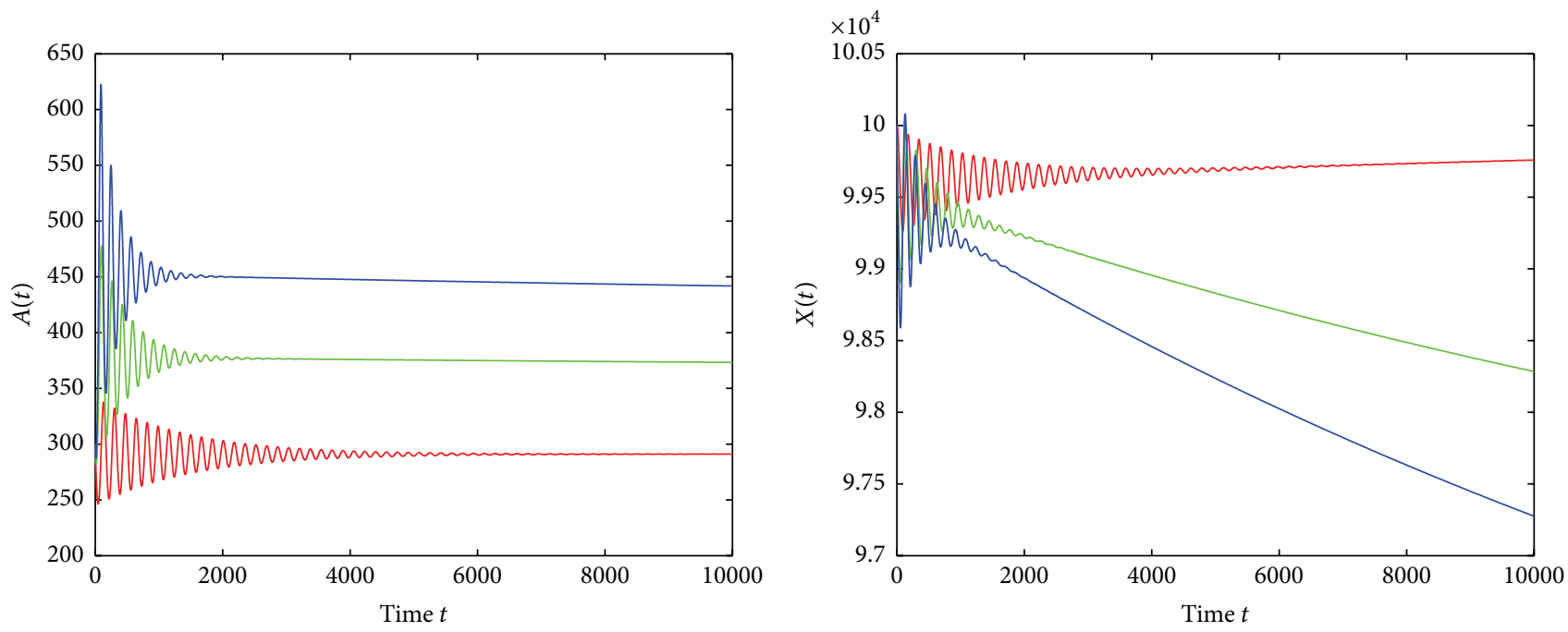

$\begin{aligned} \lambda & =0.02 \\ \lambda & =0.01 \\ \lambda & =0.005\end{aligned}$

$\lambda=0.02$
$\lambda=0.01$

$-\lambda=0.01$

$-\lambda=0.004$

(a)

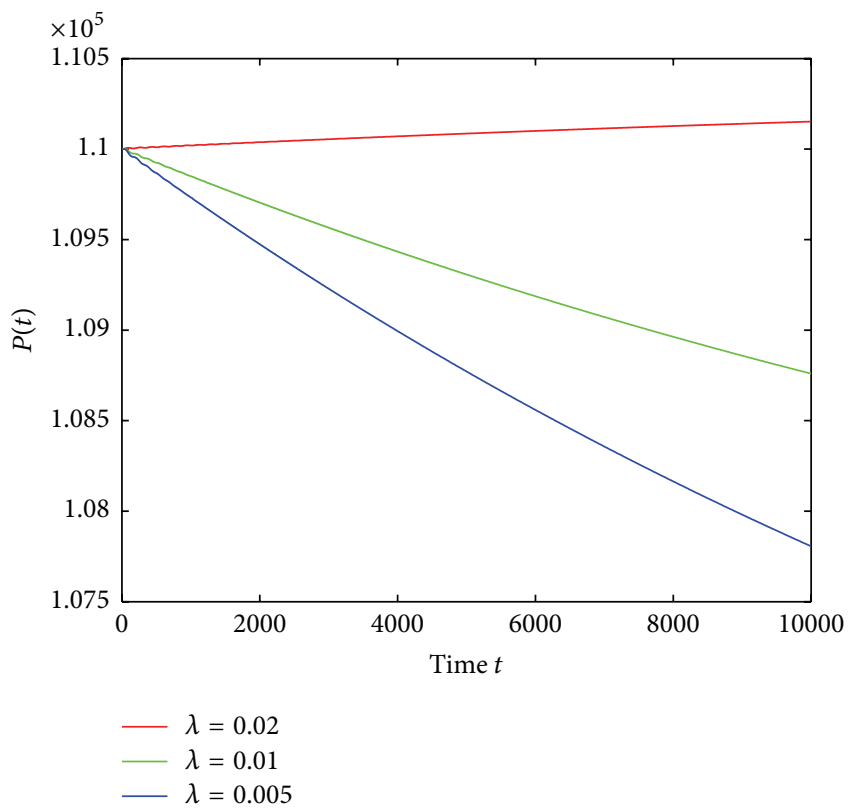

(c)
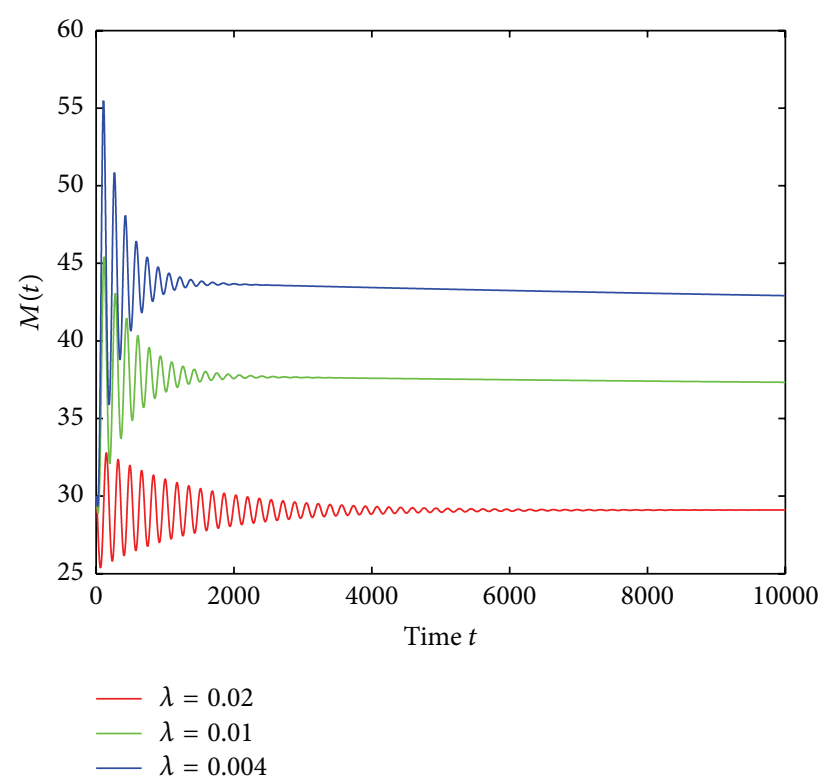

(b)

FIgURE 3: Trajectories of populations and awareness program changes for different values of $\lambda$.

By simple computation, the characteristic equation of the linearized system (22) is rewritten as follows:

$$
\begin{aligned}
\psi^{4}+ & c_{1} \psi^{3}+c_{2} \psi^{2}+c_{3} \psi+c_{4} \\
& +\left(d_{1} \psi^{3}+d_{2} \psi^{2}+d_{3} \psi+d_{4}\right) e^{-\psi \tau}=0
\end{aligned}
$$

where

$$
\begin{aligned}
& c_{1}=-b_{22}-b_{33}-b_{44}, \\
& c_{2}=b_{11} b_{22}+b_{11} b_{33}+b_{22} b_{33}+b_{22} b_{44}+b_{33} b_{44},
\end{aligned}
$$

$$
\begin{aligned}
c_{3}= & -b_{11} b_{22} b_{33}-b_{11} b_{22} b_{44}-b_{11} b_{33} b_{44}-b_{22} b_{33} b_{44}, \\
c_{4}= & b_{11} b_{22} b_{33} b_{44}, \\
d_{1}= & -c_{11}, \\
d_{2}= & b_{44} c_{11}-b_{21} c_{12}-b_{31} c_{13}, \\
d_{3}= & b_{22} c_{11}+b_{33} c_{11}+b_{21} b_{33} c_{12}+b_{22} b_{31} c_{13}+b_{21} b_{44} c_{12} \\
& \quad+b_{31} b_{44} c_{13}-b_{22} b_{33} c_{11}-b_{24} b_{41} c_{12}-b_{31} b_{23} c_{12},
\end{aligned}
$$




$$
\begin{aligned}
d_{4}= & b_{24} b_{33} b_{41} c_{12}+b_{22} b_{33} b_{44} c_{11}+b_{23} b_{31} b_{44} c_{12} \\
& -b_{22} b_{44} c_{11}-b_{33} b_{44} c_{11}-b_{22} b_{44} b_{31} c_{13} \\
& -b_{21} b_{33} b_{44} c_{12} .
\end{aligned}
$$

Now, we will derive the conditions for the stability of $E_{1}$ and the occurrence of Hopf bifurcation. Let $\psi=i \omega(\omega>0)$ be a root of (24). By substituting it into (24) and separating the real and imaginary parts, we can get the following equations:

$$
\begin{aligned}
& \left(d_{2} \omega^{2}-d_{4}\right) \cos (\omega \tau)+\left(d_{1} \omega^{3}-d_{3} \omega\right) \sin (\omega \tau) \\
& =\omega^{4}-c_{2} \omega^{2}+c_{4} \\
& \left(d_{1} \omega^{3}-d_{3} \omega\right) \cos (\omega \tau)+\left(d_{4}-d_{2} \omega^{2}\right) \sin (\omega \tau) \\
& =-c_{1} \omega^{3}+c_{3} \omega .
\end{aligned}
$$

Using the fact that $\sin ^{2}(\omega \tau)+\cos ^{2}(\omega \tau)=1$ and denoting $\eta=$ $\omega^{2}$, we obtain

$$
f(\eta)=\eta^{4}+e_{1} \eta^{3}+e_{2} \eta^{2}+e_{3} \eta+e_{4}=0
$$

where

$$
\begin{aligned}
& e_{1}=d_{1}^{2}-2 c_{2}, \\
& e_{2}=c_{2}^{2}+2 c_{4}+2 d_{1} d_{3}-d_{2}^{2}, \\
& e_{3}=2 d_{2} d_{4}-2 c_{2} c_{4}-d_{3}^{2}, \\
& e_{4}=c_{4}^{2}-d_{4}^{2} .
\end{aligned}
$$

If the coefficients $e_{i}(i=1,2,3,4$.) of (27) satisfy the Routh-Hurwitz criterion [41], then we do not get any positive value of $\eta$. In this case, (24) does not have any purely imaginary roots. In summary, we have the following result.

Theorem 3. If $R_{0}>1$ and the coefficients $e_{i}(i=1,2,3,4$.) of (27) satisfy the Routh-Hurwitz criterion, then the alcoholpresent equilibrium $E_{1}$ is locally asymptotically stable for all $\tau>$ 0.

Next, we consider the case that the coefficients $e_{i}$ ( $i=$ $1,2,3,4$.) of (27) do not satisfy the Routh-Hurwitz criterion. For example, since $\lim _{\eta \rightarrow+\infty} f(\eta)=+\infty$, we assume that $e_{4}<0$; that is, $f(0)=e_{4}<0$. Then, (27) has at least one positive real root, defined as $\eta_{*}$. In this case, we get (24) has a pair of purely imaginary roots; that is, $\pm i \omega_{*}\left(\omega_{*}=\sqrt{\eta_{*}}>0\right)$. Furthermore, via combining this $\omega_{*}$ with (26), we can get

$$
\tau_{j}=\frac{1}{\omega_{*}}\left\{\arctan \frac{d_{1} \omega_{*}^{7}+\left(c_{1} d_{2}-c_{2} d_{1}-d_{3}\right) \omega_{*}^{5}+\left(c_{2} d_{3}+c_{4} d_{1}-c_{1} d_{4}-c_{3} d_{2}\right) \omega_{*}^{3}+\left(c_{3} d_{4}-c_{4} d_{3}\right) \omega_{*}}{\left(d_{2}-c_{1} d_{1}\right) \omega_{*}^{6}+\left(c_{1} d_{3}+c_{3} d_{1}-c_{2} d_{2}-d_{4}\right) \omega_{*}^{4}+\left(c_{2} d_{4}+c_{4} d_{2}-c_{3} d_{3}\right) \omega_{*}^{2}-c_{4} d_{4}}+j \pi\right\}, \quad, \quad j=0,1,2, \ldots ;
$$

then $\psi= \pm i \omega_{*}$ are a pair of purely imaginary roots of (24) with $\tau=\tau_{j}(j=0,1,2, \ldots)$. Thus, we give the following assumption:

(H1) Equation (27) has at least one positive real root.
Suppose that (H1) holds. Without loss of generality, we assume that (27) has four positive real roots, which are defined by $\eta_{k}, k=1,2,3,4$. Then, we will have four positive real roots $\omega_{k}=\sqrt{\eta_{k}}, k=1,2,3,4$. Thus, we denote

$$
\begin{array}{r}
\tau_{k}^{\{j\}}=\frac{1}{\omega_{k}}\left\{\arctan \frac{d_{1} \omega_{k}^{7}+\left(c_{1} d_{2}-c_{2} d_{1}-d_{3}\right) \omega_{k}^{5}+\left(c_{2} d_{3}+c_{4} d_{1}-c_{1} d_{4}-c_{3} d_{2}\right) \omega_{k}^{3}+\left(c_{3} d_{4}-c_{4} d_{3}\right) \omega_{k}}{\left(d_{2}-c_{1} d_{1}\right) \omega_{k}^{6}+\left(c_{1} d_{3}+c_{3} d_{1}-c_{2} d_{2}-d_{4}\right) \omega_{k}^{4}+\left(c_{2} d_{4}+c_{4} d_{2}-c_{3} d_{3}\right) \omega_{k}^{2}-c_{4} d_{4}}+j \pi\right\} \\
k=1,2,3,4, j=0,1,2, \ldots
\end{array}
$$

Then, $\psi= \pm i \omega_{k}$ is a pair of purely imaginary roots of (24) with $\tau=\tau_{k}^{\{j\}}, k=1,2,3,4, j=0,1,2, \ldots$.

Define

$$
\tau_{0}=\tau_{k_{0}}^{\{0\}}=\min _{k \in\{1,2,3,4\}}\left\{\tau_{k}^{\{0\}}\right\}
$$

In order to investigate whether the Hopf bifurcation occurs or not as time delay $\tau$ increases through $\tau_{0}$, we will check whether the following transversality condition is satisfied. It is necessary to make the following assumption:

$$
\text { (H2) } f^{\prime}\left(\omega_{0}^{2}\right) \neq 0 \text {, where } \omega_{0}=\omega_{k_{0}} \text {. }
$$

Assume that (H2) holds; the following result is true.

Lemma 4. Assume that (H2) holds; then the transversality condition

$$
\operatorname{sgn}\left[\frac{\mathrm{dRe} \psi(\tau)}{\mathrm{d} \tau}\right]_{\tau=\tau_{0}}>0
$$

is satisfied. 
Proof. Differentiating the two sides of (24) with respect to $\tau$, we get

$$
\left[\frac{\mathrm{d} \psi(\tau)}{\mathrm{d} \tau}\right]^{-1}=\frac{4 \psi^{3}+3 c_{1} \psi^{2}+2 c_{2} \psi+c_{3}+\left(3 d_{1} \psi^{2}+2 d_{2} \psi+d_{3}\right) e^{-\psi \tau}-\left(d_{1} \psi^{3}+d_{2} \psi^{2}+d_{3} \psi+d_{4}\right) \tau e^{-\psi \tau}}{\left(d_{1} \psi^{3}+d_{2} \psi^{2}+d_{3} \psi+d_{4}\right) \psi e^{-\psi \tau}} .
$$

Combining (26) with (H2), we compute that

$$
\begin{aligned}
& {\left[\frac{\mathrm{dRe} \psi(\tau)}{\mathrm{d} \tau}\right]_{\psi=i \omega_{0}}^{-1}} \\
& \quad=\frac{\omega_{0}^{2} f^{\prime}\left(\omega_{0}^{2}\right)}{\omega_{0}^{2}\left[\left(d_{1} \omega_{0}^{3}-d_{3} \omega_{0}\right)^{2}+\left(d_{2} \omega_{0}^{2}-d_{4}\right)^{2}\right]} \\
& =\frac{f^{\prime}\left(\omega_{0}^{2}\right)}{\left(d_{1} \omega_{0}^{3}-d_{3} \omega_{0}\right)^{2}+\left(d_{2} \omega_{0}^{2}-d_{4}\right)^{2}} \neq 0 .
\end{aligned}
$$

Suppose

$$
\operatorname{sgn}\left[\frac{\mathrm{dRe} \psi(\tau)}{\mathrm{d} \tau}\right]_{\psi=i \omega_{0}}^{-1}<0
$$

we let $\tau<\tau_{0}$ and $\tau \rightarrow \tau_{0}$, which implies that (24) has a root $\psi(\tau)=\alpha(\tau)+i \omega(\tau)$ such that $\alpha(\tau)>0$, but this contradicts the definition of $\tau_{0}$. Hence, we obtain

$$
\operatorname{sgn}\left[\frac{\mathrm{dRe} \psi(\tau)}{\mathrm{d} \tau}\right]_{\tau=\tau_{0}}=\operatorname{sgn}\left[\frac{\mathrm{dRe} \psi(\tau)}{\mathrm{d} \tau}\right]_{\psi=i \omega_{0}}^{-1}>0 .
$$

This completes the proof of the lemma.

From the above discussion and Lemma 4, we have the following theorem.

Theorem 5. If $R_{0}>1$ and (H1) and (H2) hold, then the alcohol-present equilibrium $E_{1}$ is locally asymptotically stable for $\tau \in\left[0, \tau_{0}\right)$ and unstable for $\tau>\tau_{0}$. System (2a), (2b), (2c), and (2d) exhibits Hopf bifurcation at the equilibrium $E_{1}$ when $\tau=\tau_{0}$.

\section{Numerical Simulations}

With the help of MATLAB, some numerical results of system (2a), (2b), (2c), and (2d) are provided to substantiate the analytic results obtained in this section. We choose a set of values of parameters: $\Lambda=5 ; \beta=0.00002 ; \lambda=0.02 ; k_{0}=100$; $\sigma=0.001 ; p=1 ; \gamma=0.2 ; d=0.00004 ; \alpha=0.002 ; k=2.5$; $\mu=0.05$. It is easy to check that the basic reproduction number

$$
R_{0}=12.3738>1
$$

is satisfied and one positive root $A^{*}=291.6366$ can be obtained by solving (12). So, the alcohol-present equilibrium $E_{1}$ of system (2a), (2b), (2c), and (2d) is obtained as follows:

$$
\begin{aligned}
A^{*} & =291.6366, \\
X^{*} & =100075.5350, \\
P^{*} & =110468.1715, \\
M^{*} & =29.0637 .
\end{aligned}
$$

First, we can see that the value of $R_{0}$ increases as the value of $\beta$ or $\Lambda$ increases, which means that $\beta$ and $\Lambda$ play a key role in ensuring that the alcohol problem occurs (see Figure 2).

Second, the effect of awareness programs on the alcohol consumption behavior is demonstrated in Figure 3. It is apparent that awareness programs are an effective measure in controlling heavy drinking from this figure. We note that the aware population $X(t)$ increase whereas heavy drinkers $A(t)$ decrease with an increase in the value of dissemination rate $\lambda$ of awareness programs among susceptible population while the other parameters remain unchanged. Hence, alcohol problems should be controlled through the execution of awareness programs.

Moreover, according to the above given parameter values, the critical value of time delay $\tau_{0}=1.5767$ can be obtained by solving the corresponding expression. From Theorem 5, the corresponding waveforms are shown in Figures 4-7. That is, when $\tau=1.50<\tau_{0}=1.5767$, the alcohol-present equilibrium $E_{1}$ of system (2a), (2b), (2c), and (2d) is locally asymptotically stable (see Figures 4 and 5, resp.); and Hopf bifurcation occurs once $\tau=2.50>\tau_{0}=1.5767$ (see Figures 6 and 7 , resp.). These figures indicate that the system is stable for lower values of $\tau$ but for its higher values periodic solutions may arise and system may lose its stability.

\section{Conclusions and Discussions}

The goal of this paper is to analyze the impacts of awareness programs and time delay on the alcohol consumption behavior. A vast body of literature (see [11-35]) has been used for studying alcohol problems and describing the effects of awareness programs by media on the infectious disease or alcohol consumption behavior. However, most of these models are ODE models and do not incorporate the effects of the time delay. Based on the previous works, we establish an alcohol consumption model with awareness programs and time delay in Section 2. Compared to previous models, a key novelty of our model is that we introduce the delay in our 


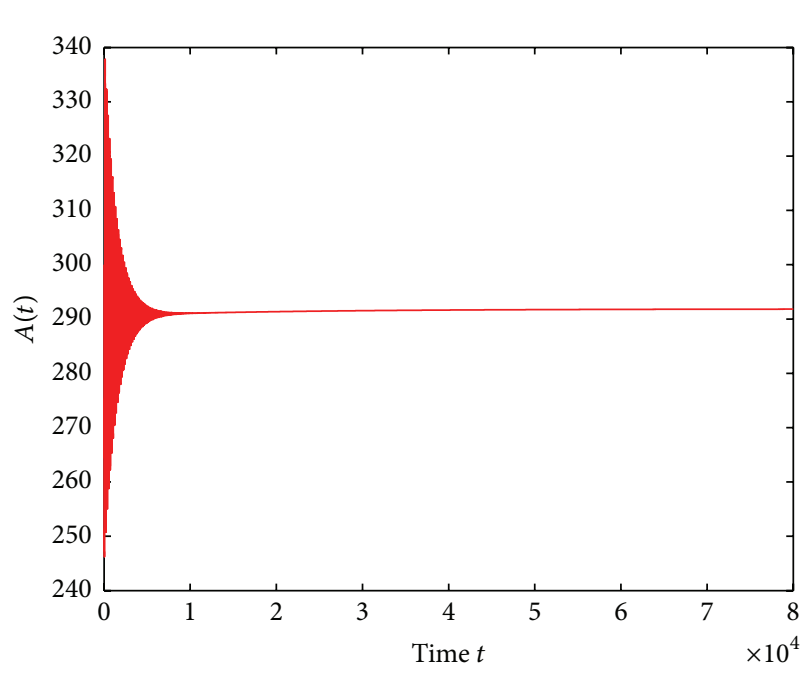

(a)

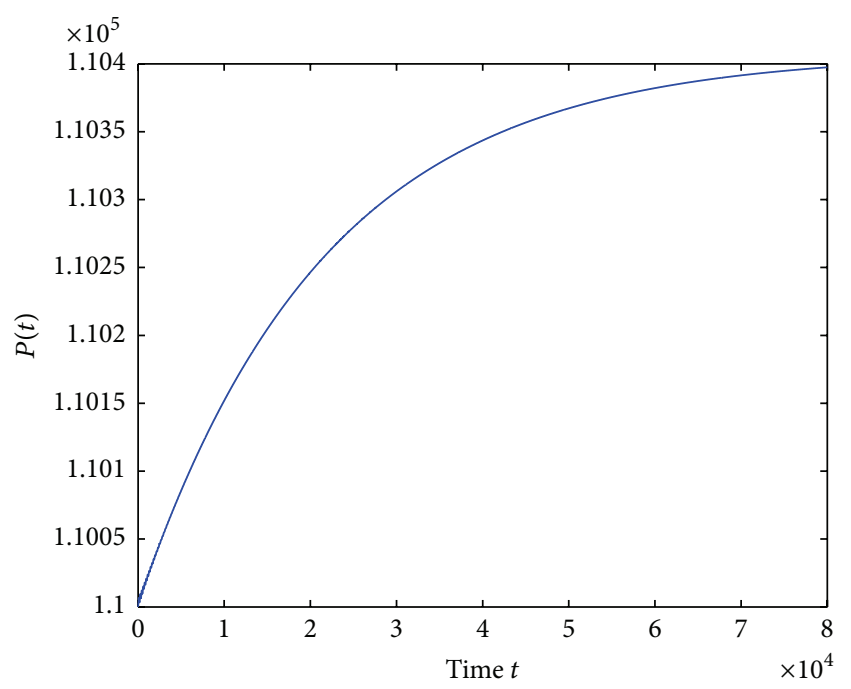

(c)

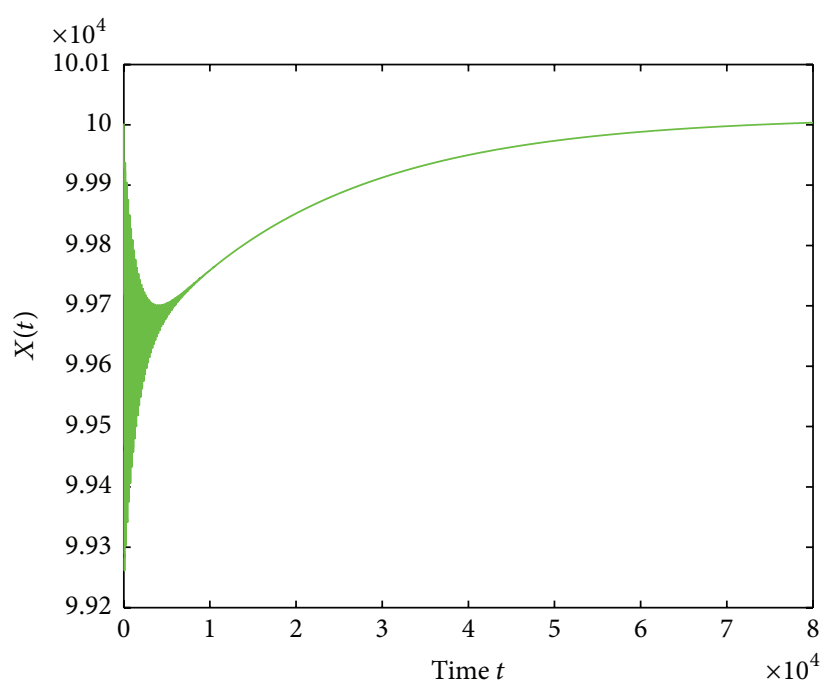

(b)

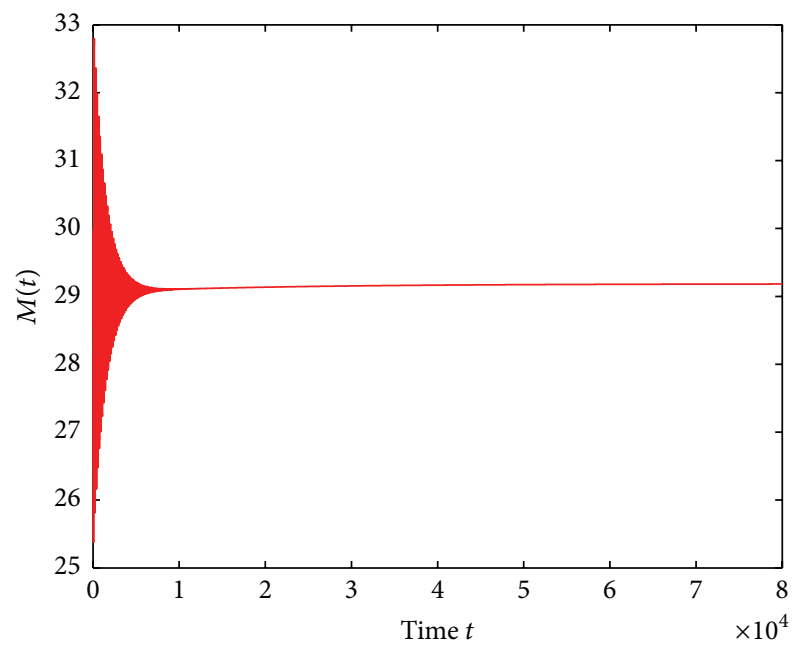

(d)

FIgURE 4: The alcohol equilibrium $E_{1}$ of system (2a), (2b), (2c), and (2d) is locally asymptotically stable with $\tau=1.50<\tau_{0}=1.5767$.

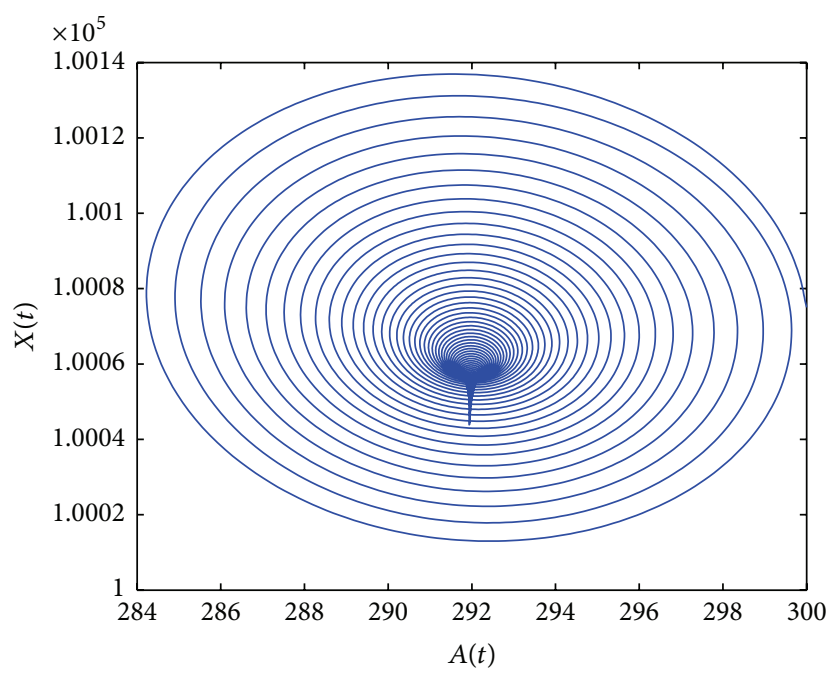

Figure 5: This corresponding phase plot of system (2a), (2b), (2c), and (2d) in $A(t)-X(t)$ plane. 


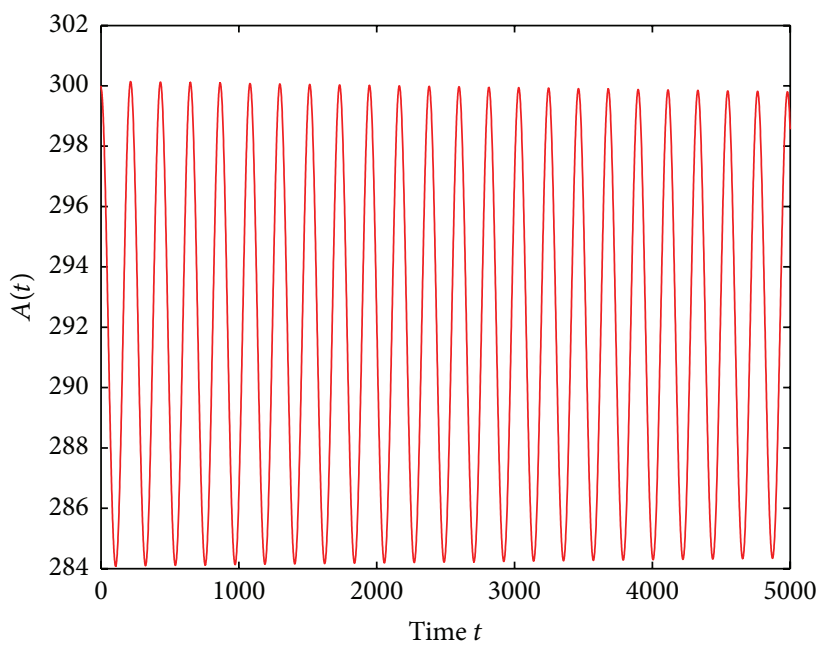

(a)

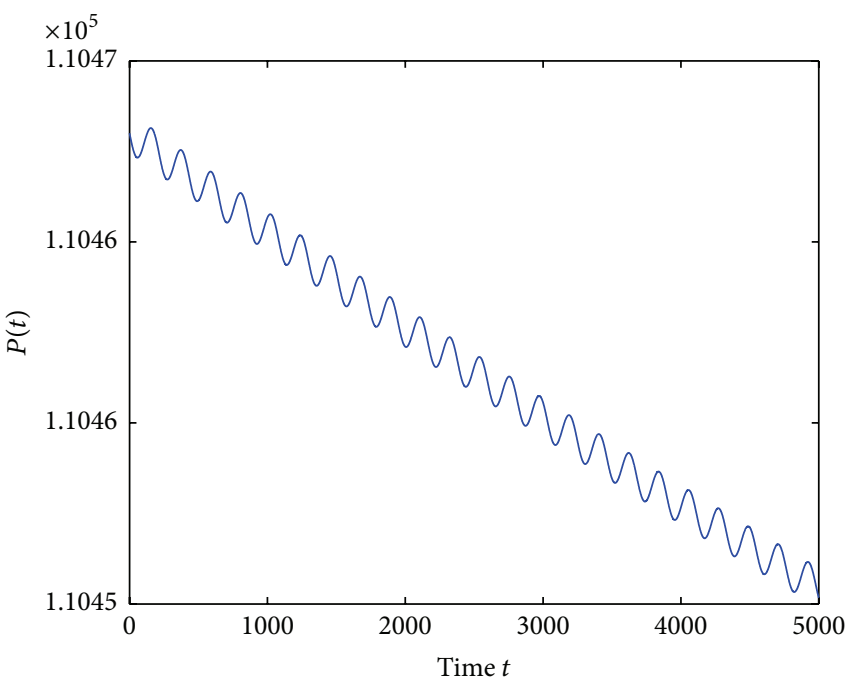

(c)

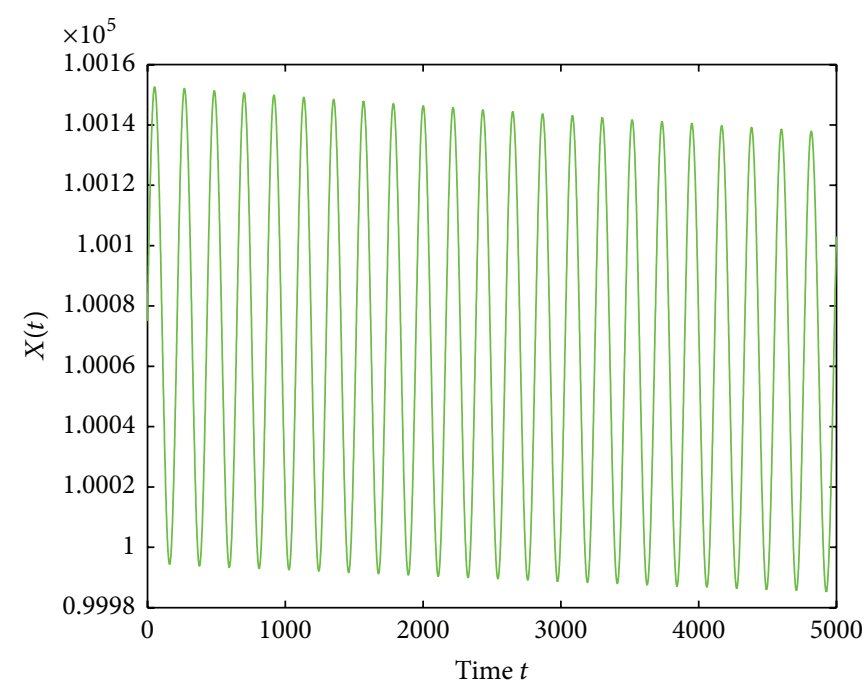

(b)

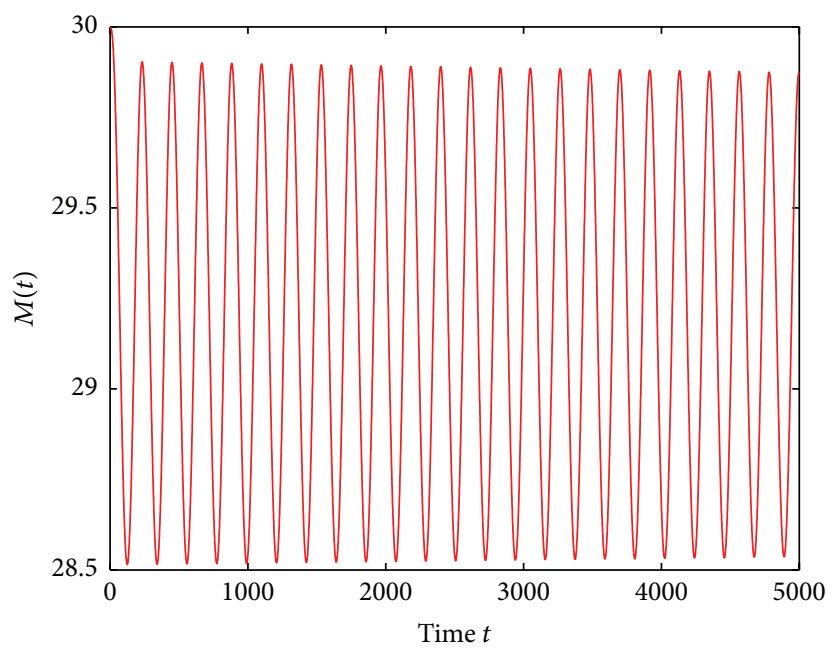

(d)

FIGURE 6: Hopf bifurcations occur with $\tau=2.50>\tau_{0}=1.5767$.

model to describe the time needed for a susceptible individual to become an infectious alcohol user. To be more realistic, we considered interaction between susceptible population and awareness programs with Holling-II functional response function. In addition, we assume that the growth rate of the cumulative density of awareness programs is proportional to the number of mortalities induced by heavy drinking. We obtain the conditions for the existence and stability of two equilibria of our model in Section 3.

At the same time, the condition of transcritical bifurcation is also given when $\tau=0$. In order to study the effect of time delay $\tau>0$ on system (2a), (2b), (2c), and (2d), we compute the linear form of system (2a), (2b), (2c), and (2d) around the alcohol-present equilibrium $E_{1}$ and analyze the local stability of $E_{1}$. In addition, we obtain the existence of Hopf bifurcation by taking time delay $\tau$ as bifurcation parameter. Our results suggest that the alcoholpresent equilibrium $E_{1}$ of system (2a), (2b), (2c), and (2d) is locally asymptotically stable for $\tau \in\left[0, \tau_{0}\right)$ and unstable for $\tau>\tau_{0}$. System (2a), (2b), (2c), and (2d) undergoes a Hopf bifurcation at the equilibrium $E_{1}$ when $\tau=\tau_{0}$. Furthermore, we study the impacts of awareness programs on the alcohol consumption behavior. Our findings show that awareness programs are an effective measure in controlling heavy drinking.

Not only awareness programs but also time delay is introduced in our model. It can help us to reduce the economic burden of disease caused by alcohol abuse. It is also beneficial to the social problems such as traffic accident and violent crime. Whether in theory or in the practical sense, these issues are very interesting and need further studies.

\section{Conflict of Interests}

The authors declare that there is no conflict of interests regarding the publication of this paper. 


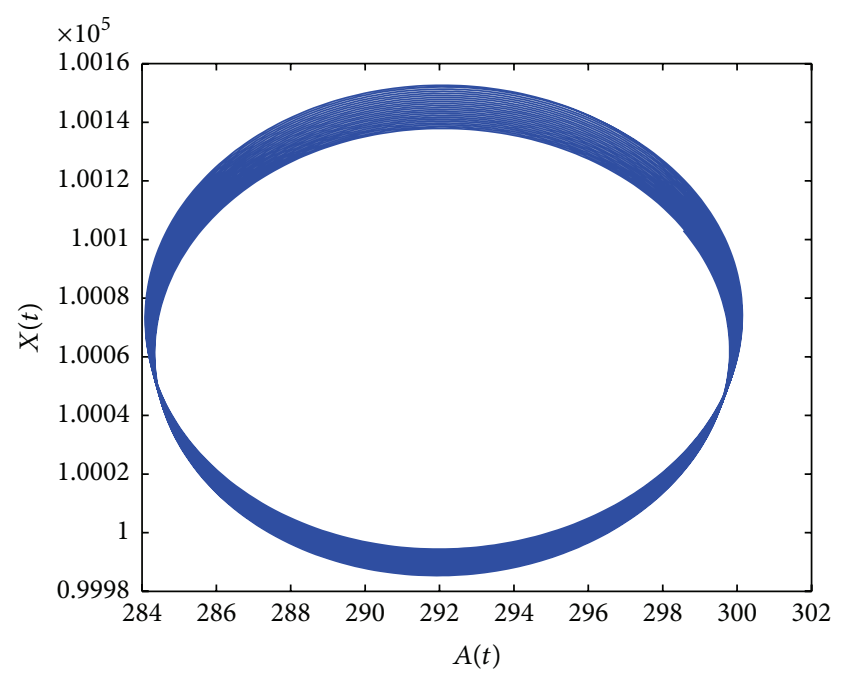

Figure 7: Periodic orbit of system (2a), (2b), (2c), and (2d) with $\tau=$ 2.50 in $A(t)-X(t)$ plane.

\section{Acknowledgments}

This work is supported by the NNSF of China (11461041), the NSF of Gansu Province (148RJZA024), and the Development Program for HongLiu Outstanding Young Teachers in Lanzhou University of Technology.

\section{References}

[1] J. Rehm, C. Mathers, S. Popova, M. Thavorncharoensap, Y. Teerawattananon, and J. Patra, "Global burden of disease and injury and economic cost attributable to alcohol use and alcohol-use disorders," The Lancet, vol. 373, no. 9682, pp. 22232233, 2009.

[2] J. Rehm, "The risk associated with alcohol use and alcoholism," Alcohol Research \& Health, vol. 34, no. 2, pp. 135-143, 2011.

[3] C.-W. Lin, C.-C. Lin, L.-R. Mo et al., "Heavy alcohol consumption increases the incidence of hepatocellular carcinoma in hepatitis B virus-related cirrhosis," Journal of Hepatology, vol. 58, no. 4, pp. 730-735, 2013.

[4] WHO, Global Status Report on Alcohol and Health 2014, WHO, Geneva, Switzerland, 2014.

[5] W. O. Kermack and A. G. McKendrick, "A contribution to the mathematical theory of epidemics," Proceedings of the Royal Society of London Series A, vol. 115, no. 772, pp. 700-721, 1927.

[6] Z. Ma, Y. Zhou, W. Wang, and Z. Jin, Mathematical Models and Dynamics of Infectious Diseases, China Sciences Press, Beijing, China, 2004.

[7] O. Sharomi and A. B. Gumel, "Curtailing smoking dynamics: a mathematical modeling approach," Applied Mathematics and Computation, vol. 195, no. 2, pp. 475-499, 2008.

[8] H.-F. Huo and C.-C. Zhu, "Influence of relapse in a giving up smoking model," Abstract and Applied Analysis, vol. 2013, Article ID 525461, 12 pages, 2013.

[9] E. White and C. Comiskey, "Heroin epidemics, treatment and ODE modelling," Mathematical Biosciences, vol. 208, no. 1, pp. 312-324, 2007.

[10] G. Mulone and B. Straughan, "A note on heroin epidemics," Mathematical Biosciences, vol. 218, no. 2, pp. 138-141, 2009.
[11] B. Benedict, "Modelling alcoholism as a contagious disease: how 'infected' drinking buddies spread problem drinking," SIAM News, vol. 40, no. 3, 2007.

[12] J. L. Manthey, A. Y. Aidoo, and K. Y. Ward, "Campus drinking: an epidemiological model," Journal of Biological Dynamics, vol. 2, no. 3, pp. 346-356, 2008.

[13] A. Mubayi, P. E. Greenwood, C. Castillo-Chávez, P. J. Gruenewald, and D. M. Gorman, "The impact of relative residence times on the distribution of heavy drinkers in highly distinct environments," Socio-Economic Planning Sciences, vol. 44, no. 1, pp. 45-56, 2010.

[14] R. Bani, R. Hameed, S. Szymanowski, P. Greenwood, C. KribsZaleta, and A. Mubayi, "Influence of environmental factors on college alcohol drinking patterns," Mathematical Biosciences and Engineering, vol. 10, no. 5-6, pp. 1281-1300, 2013.

[15] B. Buonomo and D. Lacitignola, "Modeling peer influence effects on the spread of high-risk alcohol consumption behavior," Ricerche di Matematica, vol. 63, no. 1, pp. 101-117, 2014.

[16] A. Mubayi and P. E. Greenwood, "Contextual interventions for controlling alcohol drinking," Mathematical Population Studies, vol. 20, no. 1, pp. 27-53, 2013.

[17] G. Mulone and B. Straughan, "Modeling binge drinking," International Journal of Biomathematics, vol. 5, no. 1, 14 pages, 2012.

[18] H.-F. Huo and N.-N. Song, "Global stability for a binge drinking model with two stages," Discrete Dynamics in Nature and Society, vol. 2012, Article ID 829386, 15 pages, 2012.

[19] C. P. Bhunu and S. Mushayabasa, "A theoretical analysis of smoking and alcoholism," Journal of Mathematical Modelling and Algorithms, vol. 11, no. 4, pp. 387-408, 2012.

[20] C. E. Walters, B. Straughan, and J. R. Kendal, "Modelling alcohol problems: total recovery," Ricerche di Matematica, vol. 62, no. 1, pp. 33-53, 2013.

[21] X. Y. Wang, H. F. Huo, Q. K. Kong, and W. X. Shi, "Optimal control strategies in an alcoholism model," Abstract and Applied Analysis, vol. 2014, Article ID 954069, 18 pages, 2014.

[22] S. Del Valle, A. M. Evangelista, M. C. Velasco, C. M. KribsZaleta, and S.-F. H. Schmitz, "Effects of education, vaccination and treatment on HIV transmission in homosexuals with genetic heterogeneity," Mathematical Biosciences, vol. 187, no. 2, pp. 111-133, 2004.

[23] R. Liu, J. Wu, and H. Zhu, "Media/psychological impact on multiple outbreaks of emerging infectious diseases," Computational and Mathematical Methods in Medicine, vol. 8, no. 3, pp. 153$164,2007$.

[24] F. Nyabadza, C. Chiyaka, Z. Mukandavire, and S. D. HoveMusekwa, "Analysis of an HIV/AIDS model with public-health information campaigns and individual withdrawal," Journal of Biological Systems, vol. 18, no. 2, pp. 357-375, 2010.

[25] Y. Liu and J.-A. Cui, "The impact of media coverage on the dynamics of infectious disease," International Journal of Biomathematics, vol. 1, no. 1, pp. 65-74, 2008.

[26] J.-A. Cui, X. Tao, and H. Zhu, "An SIS infection model incorporating media coverage," Rocky Mountain Journal of Mathematics, vol. 38, no. 5, pp. 1323-1334, 2008.

[27] J. Cui, Y. Sun, and H. Zhu, "The impact of media on the control of infectious diseases," Journal of Dynamics and Differential Equations, vol. 20, no. 1, pp. 31-53, 2008.

[28] C. Sun, W. Yang, J. Arino, and K. Khan, "Effect of mediainduced social distancing on disease transmission in a two patch setting," Mathematical Biosciences, vol. 230, no. 2, pp. 87-95, 2011. 
[29] I. Z. Kiss, J. Cassell, M. Recker, and P. L. Simon, "The impact of information transmission on epidemic outbreaks," Mathematical Biosciences, vol. 225, no. 1, pp. 1-10, 2010.

[30] S. Funk, E. Gilad, and V. A. A. Jansen, "Endemic disease, awareness, and local behavioural response," Journal of Theoretical Biology, vol. 264, no. 2, pp. 501-509, 2010.

[31] S. Samanta, S. Rana, A. Sharma, A. K. Misra, and J. Chattopadhyay, "Effect of awareness programs by media on the epidemic outbreaks: a mathematical model," Applied Mathematics and Computation, vol. 219, no. 12, pp. 6965-6977, 2013.

[32] Y. N. Xiao, T. T. Zhao, and S. Y. Tang, "Dynamics of an infectious diseases with media/psychology induced non-smooth incidence," Mathematical Biosciences and Engineering, vol. 10, no. 2, pp. 445-461, 2013.

[33] A. K. Misra, A. Sharma, and J. B. Shukla, "Modeling and analysis of effects of awareness programs by media on the spread of infectious diseases," Mathematical and Computer Modelling, vol. 53, no. 5-6, pp. 1221-1228, 2011.

[34] A. K. Misra, A. Sharma, and V. Singh, "Effect of awareness programs in controlling the prevalence of an epidemic with time delay," Journal of Biological Systems, vol. 19, no. 2, pp. 389402, 2011.

[35] H.-F. Huo and Q. Wang, "Modelling the influence of awareness programs by media on the drinking dynamics," Abstract and Applied Analysis, vol. 2014, Article ID 938080, 8 pages, 2014.

[36] Y. Kuang, Delay Differential Equations with Application in Population Dynamics, vol. 191 of Mathematics in Science and Engineering, Academic Press, Boston, Mass, USA, 1993.

[37] J. Li and Y. Kuang, "Analysis of a model of the glucose-insulin regulatory system with two delays," SIAM Journal on Applied Mathematics, vol. 67, no. 3, pp. 757-776, 2007.

[38] J. Li, M. Wang, P. Palumbo, S. Panunzi, and A. De Gaetano, "The range of time delay and the global stability of the equilibrium for an IVGTT model," Mathematical Biosciences, vol. 235, no. 2, pp. 128-137, 2012.

[39] C. Castillo-Chavez and B. Song, "Dynamical models of tuberculosis and their applications," Mathematical Biosciences and Engineering, vol. 1, no. 2, pp. 361-404, 2004.

[40] P. van den Driessche and J. Watmough, "Reproduction numbers and sub-threshold endemic equilibria for compartmental models of disease transmission," Mathematical Biosciences, vol. 180, pp. 29-48, 2002.

[41] M. Kot, Elements of Mathematical Biology, Cambridge University Press, Cambridge, UK, 2001.

[42] N. Chitnis, J. M. Hyman, and J. M. Cushing, "Determining important parameters in the spread of malaria through the sensitivity analysis of a mathematical model," Bulletin of Mathematical Biology, vol. 70, no. 5, pp. 1272-1296, 2008. 


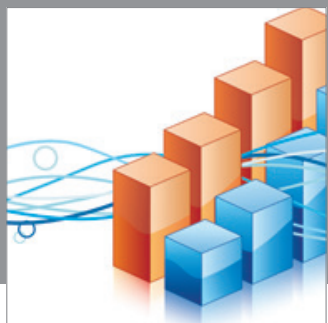

Advances in

Operations Research

mansans

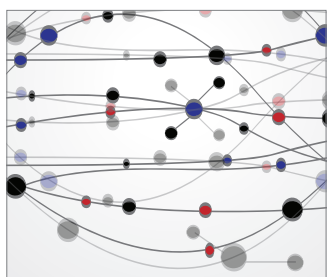

The Scientific World Journal
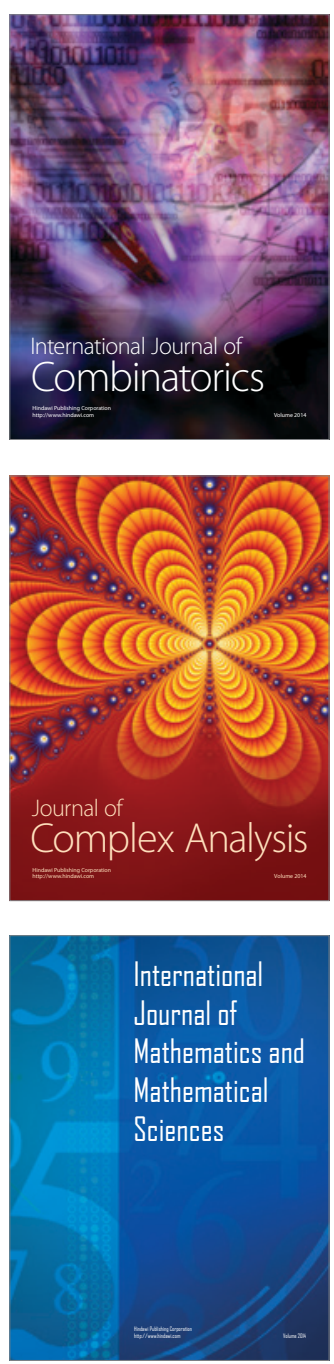
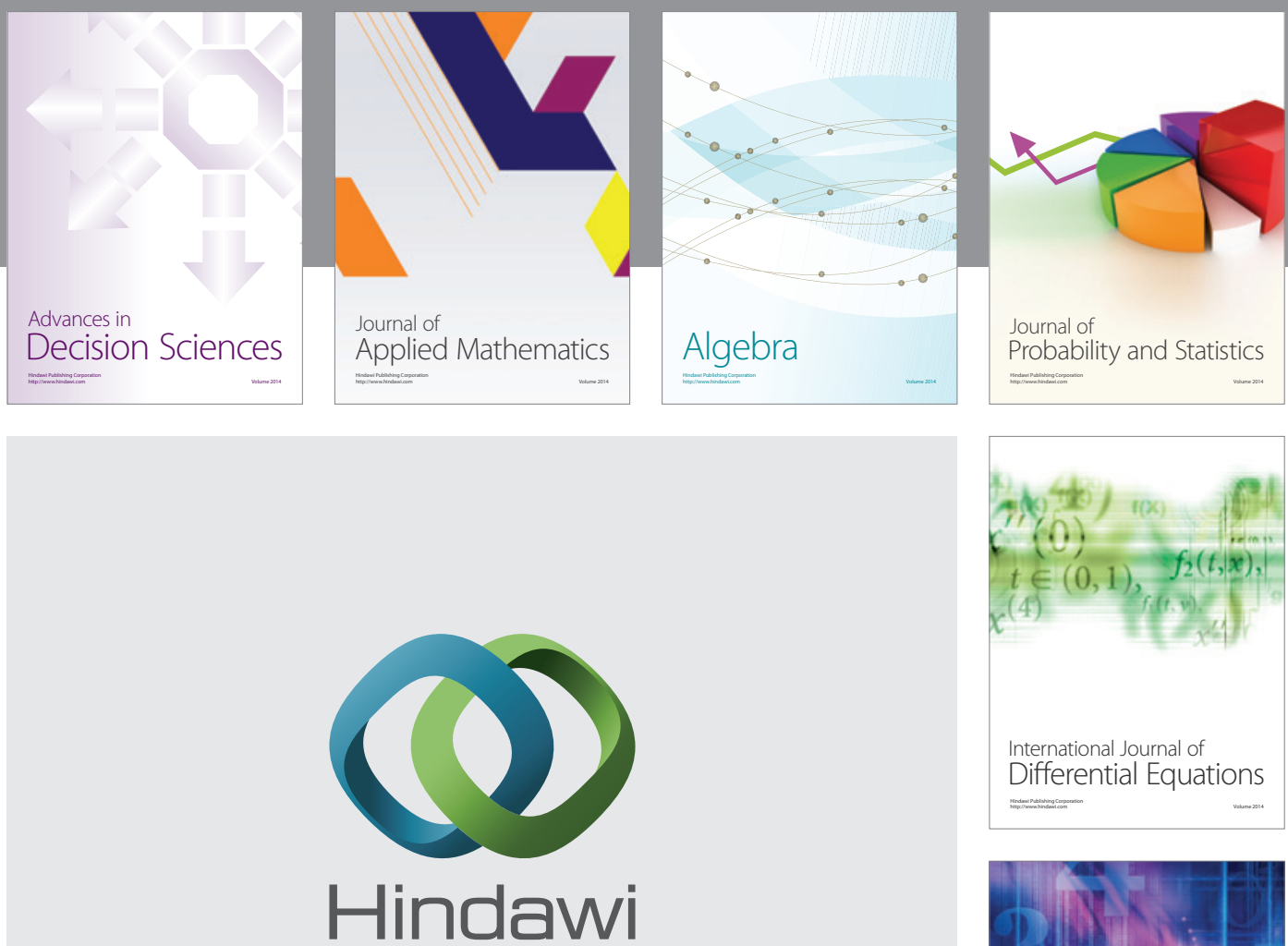

Submit your manuscripts at http://www.hindawi.com
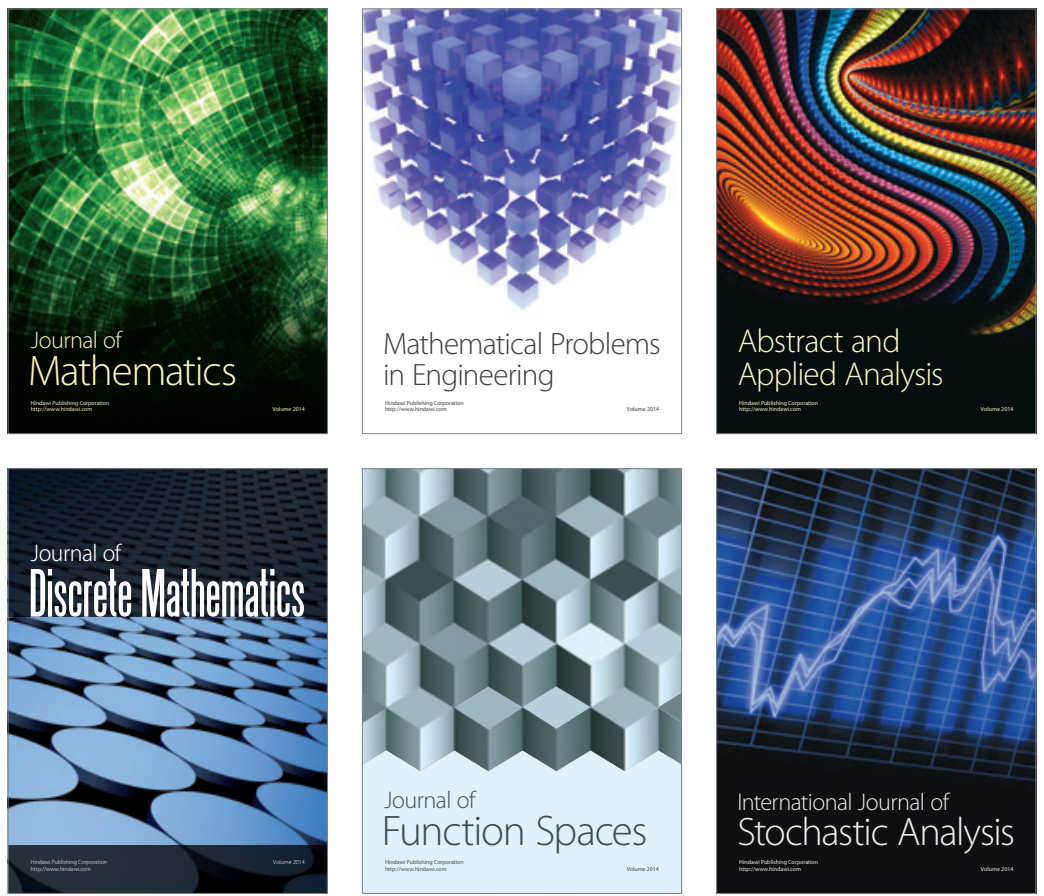

Journal of

Function Spaces

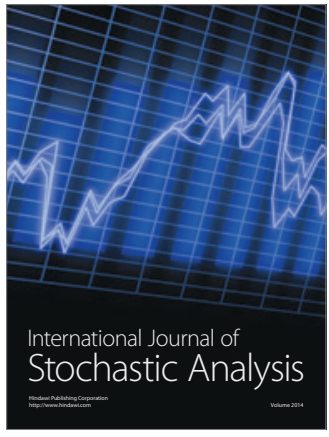

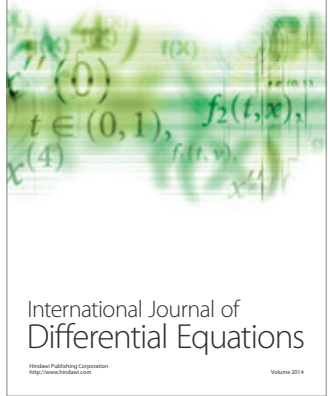
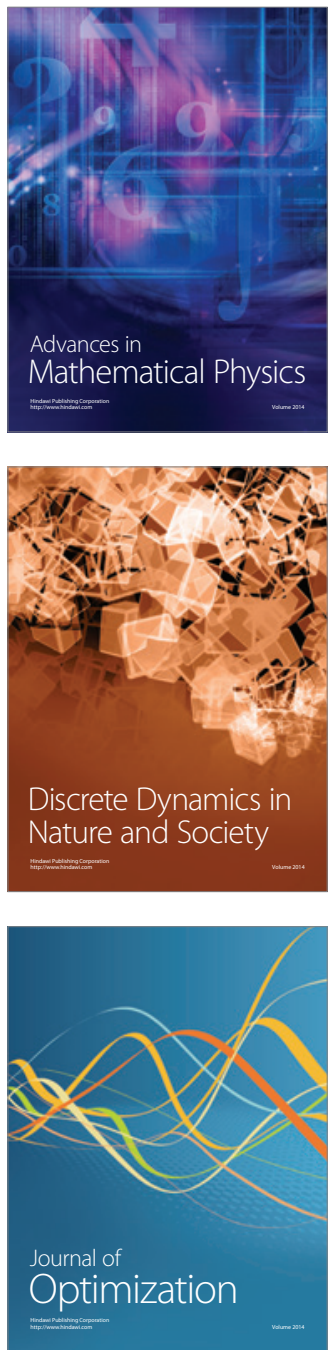